\title{
ADF/cofilin proteins translocate to mitochondria during apoptosis but are not generally required for cell death signaling
}

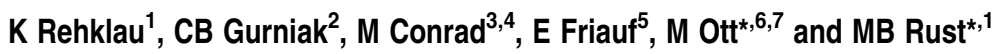

Non-muscle cofilin ( $n$-cofilin) is a member of the ADF/cofilin family of actin depolymerizing proteins. Recent studies reported a mitochondrial translocation of $n$-cofilin during apoptosis. As these studies also revealed impaired cytochrome $c$ release and a block in apoptosis upon small interfering RNA-mediated n-cofilin knockdown, n-cofilin was postulated to be essential for apoptosis induction. To elucidate the general importance of ADF/cofilin activity for apoptosis, we exposed mouse embryonic fibroblasts deficient for $n$-cofilin, ADF (actin depolymerizing factor), or all ADF/cofilin isoforms to well-characterized apoptosis inducers. Cytochrome $c$ release, caspase- 3 activation, and apoptotic chromatin condensation were unchanged in all mutant fibroblasts. Thus, we conclude that ADF/cofilin activity is not generally required for induction or progression of apoptosis in mammalian cells. Interestingly, mitochondrial association of ADF and n-cofilin during apoptosis was preceded by, and dependent on, actin that translocated by a yet unknown mechanism to mitochondria during cell death.

Cell Death and Differentiation (2012) 19, 958-967; doi:10.1038/cdd.2011.180; published online 2 December 2011

Apoptosis is essential for many fundamental processes, such as embryogenesis and tissue homeostasis. Consequently, several human diseases, including cancer and neurodegenerative disorders, are caused by defects in this highly regulated form of cell death. ${ }^{1,2}$ Mitochondria are crucial for the execution of apoptosis. ${ }^{3}$ Pro-apoptotic proteins, such as cytochrome $c$ and second mitochondria-derived activator of caspase (SMAC), are released from mitochondria into the cytosol where they participate in the activation of execution caspases. These proteases are responsible for the dismantling of cells during apoptosis. ${ }^{4}$ Although the mechanisms of caspase activation in the cytosol are well characterized, the steps that control cytochrome $c$ release from mitochondria and thereby activate the apoptosis program have remained largely elusive.

Outer mitochondrial membrane permeabilization and cytochrome $c$ release are typically achieved by pro-apoptotic $\mathrm{Bcl}-2$ proteins, such as Bax and Bak. The release of cytochrome $c$ can be modulated by various factors, such as changes in the cellular redox balance and lipid homeostasis, mitochondrial dynamics, and perturbations in the cytoskeleton. ${ }^{5-7}$ Specifically, an important role of actin dynamics for apoptosis signaling was recently suggested. ${ }^{5}$ Actin dynamics critically depend on members of the ADF/cofilin family that comprises muscle cofilin (m-cofilin), non-muscle cofilin (n-cofilin), and ADF (actin depolymerizing factor). ${ }^{8}$ Recently, it was shown that $\mathrm{n}$-cofilin translocates to mitochondria during the early phase of apoptosis, but the mechanism for this translocation remained obscure. ${ }^{9-12}$ Interestingly, when n-cofilin expression was decreased by small interfering RNA (siRNA)mediated gene knockdown, cytochrome $c$ release and apoptosis execution were strongly inhibited. ${ }^{9,10}$ It was therefore postulated that mitochondrial translocation of $n$-cofilin is an essential step in the induction of apoptosis that links actin dynamics to mitochondrial function. ${ }^{9,13}$

To test the relevance of ADF/cofilin activity for apoptotic processes, we here employed a genetically well-defined system of mouse embryonic fibroblasts (MEFs) devoid of any ADF/cofilin activity and challenged these cells with wellcharacterized apoptosis inducers. We found that, upon induction of apoptosis in control MEFs, n-cofilin, and its homolog ADF translocated to mitochondria together with actin. Interestingly, our data suggest no direct interaction of $\mathrm{n}$-cofilin and ADF with mitochondria, but a rather indirect, actin-mediated association. Importantly, the mitochondrial association of $\mathrm{n}$-cofilin and ADF had no impact on apoptosis

${ }^{1}$ Neurobiology/Neurophysiology Group, Department of Biology, University of Kaiserslautern, Kaiserslautern, Germany; ${ }^{2}$ Department of Mathematics and Natural Sciences, Institute of Genetics, University of Bonn, Bonn, Germany; ${ }^{3}$ German Center for Neurodegenerative Diseases (DZNE), Munich, Germany; ${ }^{4}$ Institute of Developmental Genetics, Helmholtz Zentrum München, Munich, Germany; ${ }^{5}$ Animal Physiology Group, Department of Biology, University of Kaiserslautern, Kaiserslautern, Germany; ${ }^{6}$ Membrane Biogenesis Group, Department of Biology, University of Kaiserslautern, Kaiserslautern, Germany and ${ }^{7}$ Department of Biochemistry and Biophysics, Center for Biomembrane Research, Stockholm University, Stockholm, Sweden

${ }^{*}$ Corresponding authors: MB Rust, Neurobiology/Neurophysiology Group, Department of Biology, University of Kaiserslautern, 67663 Kaiserslautern, Germany.

Tel: + 49631205 4669; Fax: 49631205 4684; E-mail: marco.rust@ biologie.uni-kl.de

or M Ott, Department of Biochemistry and Biophysics, Center for Biomembrane Research, Stockholm University, 10691 Stockholm, Sweden. Tel: + 46 8162595 ;

Fax: + 468 155597; E-mail: martin.ott@dbb.su.se

Keywords: cofilin; actin; mitochondria; apoptosis; cytochrome $c$

Abbreviations: 3T3, 3-day transfer protocol; AC-MEF, MEF-deficient for ADF and n-cofilin; ADF, actin-depolymerizing factor; COX II, cytochrome $c$ oxidase subunit II; E, embryonic; flx, floxed; JAS, jasplakinolide; MCM, OH-TAM-inducible cre recombinase; m-cofilin, muscle cofilin; MEF, mouse embryonic fibroblast; MSH buffer, mannitol-sucrose-hepes-buffer; $n$-Cof ${ }^{-I-}$ MEF, MEFs deficient for $n$-cofilin; $n$-Cofflyfilx MEF, MEFs with floxed $n$-cofilin gene; $n$-cofilin, non-muscle cofilin; OH-TAM, 4-hydroxytamoxifen; P, passage; PGAM, phosphoglycerate mutase; PHAL, phalloidin; phospho-AC, phosphorylated ADF/cofilin; siRNA, small interfering RNA; SMAC, second mitochondria-derived activator of caspase; STS, staurosporine; WT, wild type

Received 30.5.11; revised 31.10.11; accepted 31.10.11; Edited by L Scorrano; published online 02.12.11 
signaling because ablation of either $n$-cofilin, ADF, or both proteins together did not change cell death progression. In summary, our work demonstrates that in mammalian cells ADF/cofilin activity is not generally required for efficient induction of apoptosis.

\section{Results and Discussion}

$\mathrm{N}$-cofilin and ADF translocate to mitochondria in MEFs upon exposure to staurosporine. In this study, we set out to characterize the role of ADF/cofilin activity for the induction and progression of apoptosis using MEFs as model cells. These cells contain substantial amounts of both n-cofilin and ADF (Figure 1a). We first asked whether these two actin depolymerizing proteins translocate to mitochondria when MEFs undergo apoptosis. We exposed MEFs to staurosporine (STS), a nonspecific kinase inhibitor that provokes apoptosis in most cell types including MEFs, and analyzed the subcellular distribution of $n$-cofilin and ADF by cell fractionation and western blotting. After $2 \mathrm{~h}$ of STS exposure, the pro-apoptotic protein Bax translocated to mitochondria, whereas cytochrome $c$ was released into the cytosol (Figure 1b). N-cofilin and ADF were absent from the mitochondrial fraction of untreated MEFs, but both proteins co-fractionated with mitochondria after $2 \mathrm{~h}$ of STS exposure. To prove that co-migration of $n$-cofilin and ADF indeed reflects a tight association with mitochondria and is no artifact introduced by fractionation via differential centrifugation, we tested whether these proteins would co-migrate with mitochondria in floatation gradients. In this setup, particles are fractionated depending on their density, and mitochondria float to the sucrose density that reflects their own density (Figure 1c, left). Consequently, proteins associated with mitochondria show identical fractionation. When testing for co-fractionation of $n$-cofilin and ADF, both proteins indeed associated with mitochondria (Figure 1c, right).

a

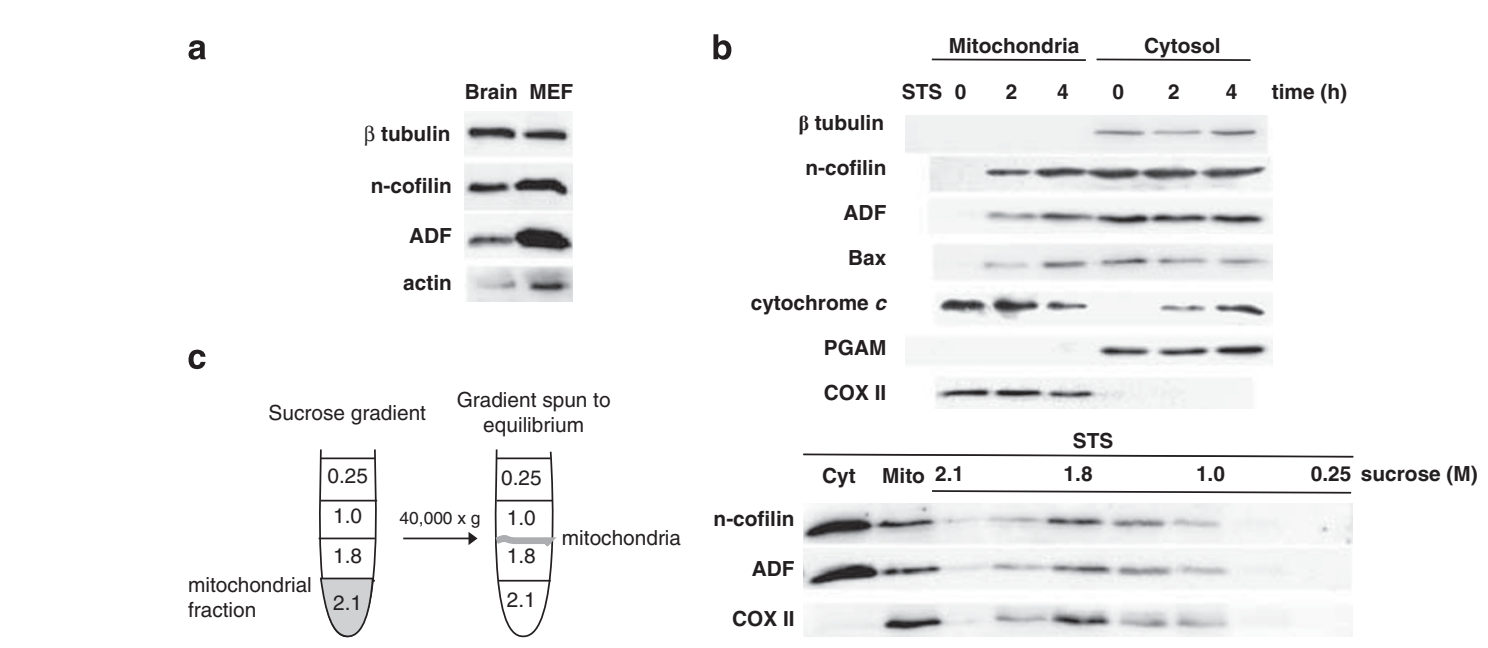

b

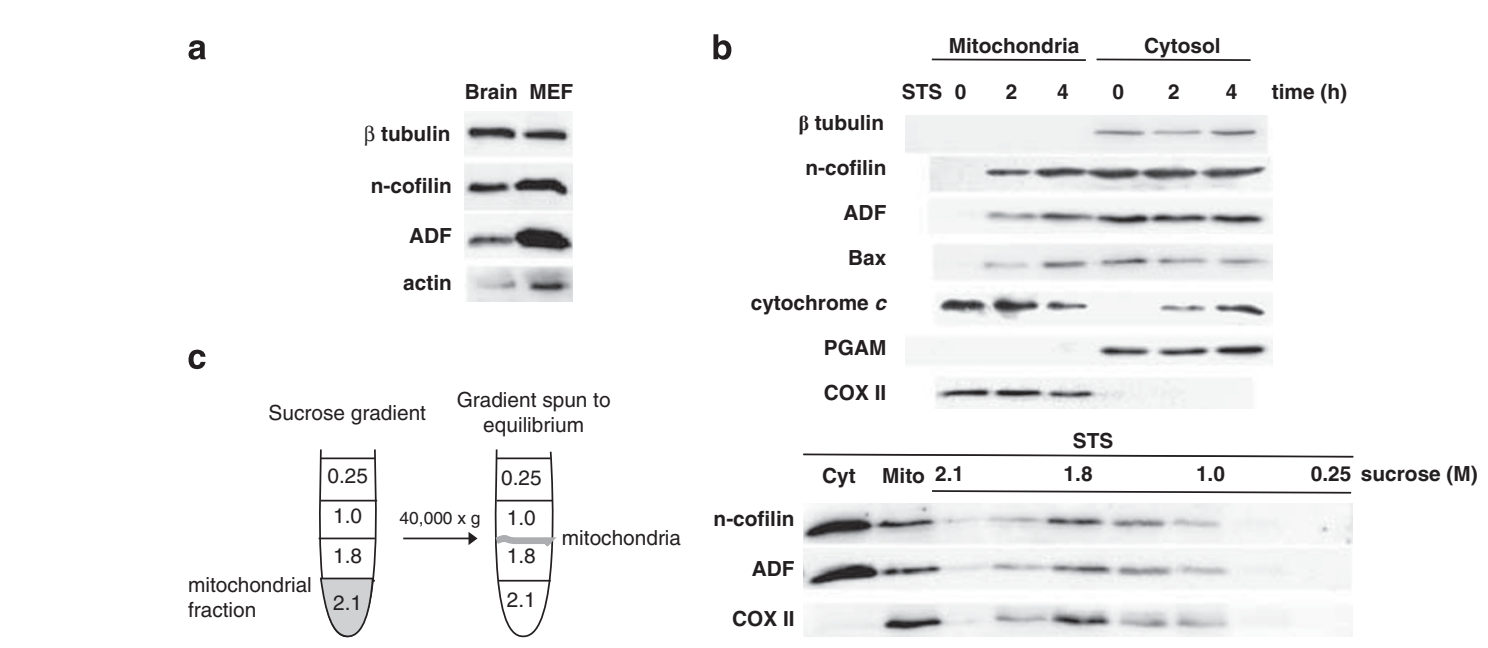

Preparation of MEFs lacking ADF, n-cofilin, or both proteins. Having demonstrated a mitochondrial translocation of n-cofilin and ADF during apoptosis in MEFs, we next set out to characterize the role of $n$-cofilin and ADF for apoptosis signaling. Because both proteins share very similar biochemical properties ${ }^{8}$ and were suggested to functionally replace each other, ${ }^{14,15}$ we investigated apoptotic responses upon ablation of $n$-cofilin, ADF, or both proteins. To avoid the well-known problems associated with siRNA-mediated knockdown, we chose a clean genetic strategy and prepared MEF cultures from conditional n-cofilin-knockout embryos $\left(n-C^{f} f^{f / x / f l x}\right)$, ADF-null embryos $\left(A D F^{-/}\right)$, or $A D F^{-/} / n-C^{f} f^{f / x / x}$ embryos. ${ }^{16}$ Because systemic deletion of $n$-Cof in mice is embryonically lethal, ${ }^{17}$ we disrupted the $n$-cofilin gene in cell culture. To this end, we transfected MEFs from $n$-Cof $f^{f / x / f I x}$ and $A D F^{-/-} / n-C_{0} f^{f / x / f l x}$ mice with a plasmid encoding a variant of the Cre recombinase (OH-TAM-inducible cre recombinase; $\mathrm{MCM}$ ), which can be activated by 4-hydroxytamoxifen (OH-TAM) treatment ${ }^{18}$ (Figures $2 a$ and b). Exposure of the transfected cells to $\mathrm{OH}-\mathrm{TAM}$ resulted in a complete deletion of the $n$-cofilin gene in MEFs from $n-C^{f f l x / f l x}$ and $A D F^{-1} / n-C^{f} f^{f \mid x / f l x}$ mice (Figure $2 \mathrm{c}$ ). At $72 \mathrm{~h}$ after $\mathrm{OH}$-TAM treatment, no $\mathrm{n}$-cofilin signal was detectable by western blotting (Figure 2d), demonstrating successful protein depletion in MEFs. Next, we compared expression of ADF/cofilin proteins in the different mutant MEF lines (Figure 2e) and found unchanged ADF levels in n-cofilindeficient MEFs ( $n$-Cof $f^{f / x / f l x}$ MEFs) as well as unchanged $n$ cofilin levels in MEFs from $A D F^{-1-}$ mice. Importantly, by using an antibody that recognizes $n$-cofilin and the musclespecific isoform m-cofilin, we found that m-cofilin was not expressed in MEFs and that it was not activated in the absence of $n$-cofilin and ADF. Hence, our $A D F^{-1-} / n-C^{-1-}$ MEFs (MEFs deficient for ADF and n-cofilin; AC-MEFs) were devoid of any ADF/cofilin protein and thus represent an ideal system to investigate the relevance of ADF/cofilin activity for apoptotic processes.

Figure $1 \mathrm{~N}$-cofilin and ADF translocate to mitochondria in MEFs upon exposure to STS. (a) N-cofilin and ADF are expressed in MEFs. Lysates of MEFs or mouse brain were analyzed by western blotting using the indicated antibodies. Equal loading was controlled by analyzing levels of $\beta$-tubulin. (b) Translocation of n-cofilin and ADF to mitochondria during STS-evoked apoptosis. Induction of apoptosis was confirmed by cytochrome $c$ release and by Bax translocation. Untreated and STS-treated MEFs were harvested and separated into a cytosol (cytosolic marker: PGAM) and a mitochondrial fraction (mitochondrial marker COX II). (c) N-cofilin and ADF are associated to mitochondria. The mitochondrial fraction of STS-treated MEFs was fractionated using floatation centrifugation (left). Western blot analysis of the gradient fractions revealed co-migration of $n$-cofilin and ADF with mitochondria 
a

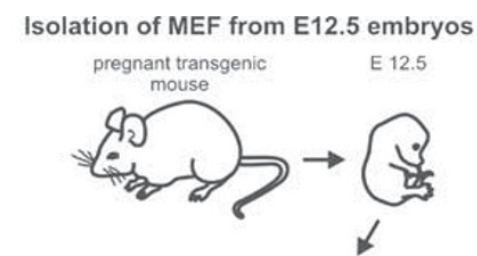

Transfection with OH-TAM-inducible Cre (MCM)

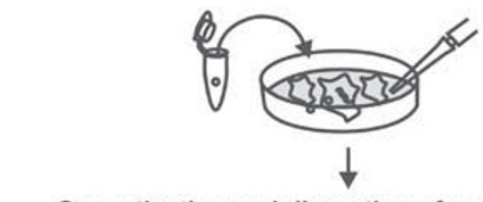

Cre activation and disruption of $n$-cofilin gene

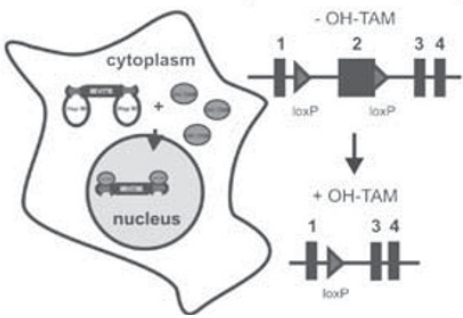

d

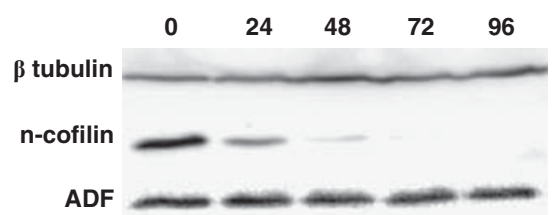

b

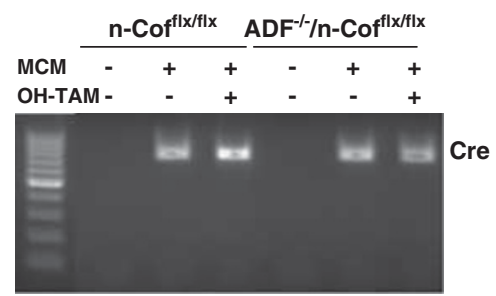

C
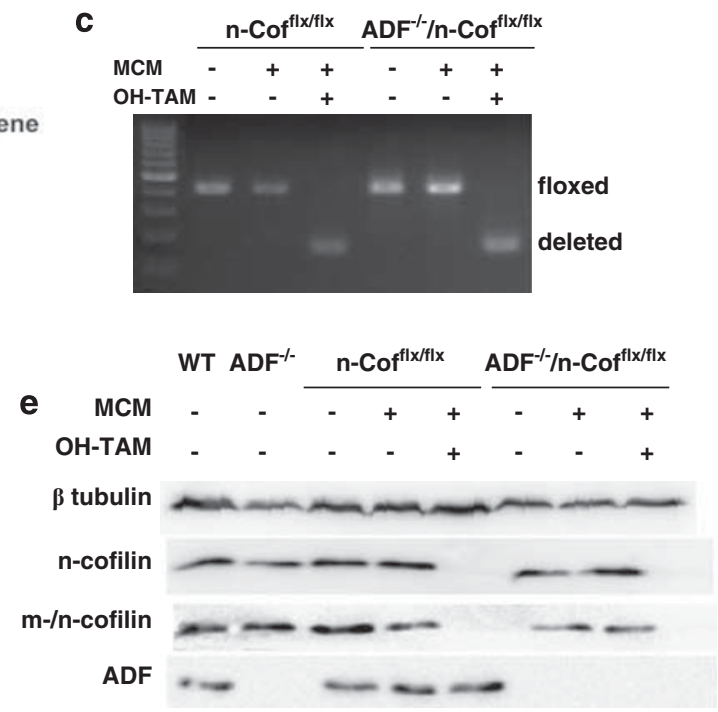

Figure 2 Preparation of MEFs lacking ADF, n-cofilin, or both proteins. (a) Experimental outline for the generation of ADF- and/or n-cofilin-deficient MEFs. MEFs were isolated from E12.5 embryos and passaged until spontaneous immortalization. Disruption of the floxed $n$-cofilin gene was obtained by stable transfection of MEFs with MCM and subsequent OH-TAM treatment. (b) PCR results confirming the expression of MCM in transfected $n-$ Cof $^{f / x f l x}$ and $A D F^{-1} / n-C$ of $f^{\text {Ixflx }}$ MEFs. (c) PCR results confirming efficient disruption of the n-cofilin gene (deleted) in MCM-transfected and OH-TAM-treated MEFs. In contrast, the $n$-cofilin gene is undisrupted (floxed) in non-transfected

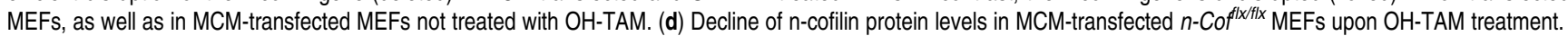
At $72 \mathrm{~h}$ after the onset of OH-TAM treatment, $\mathrm{n}$-cofilin protein was undetectable. ADF expression was unaltered in $\mathrm{n}$-cofilin-deficient MEFs; $\beta$-tubulin was used to control protein loading. (e) Characterization of the four different MEF lines. No change in n-cofilin protein level was detected upon transfection with MCM. An antibody recognizing

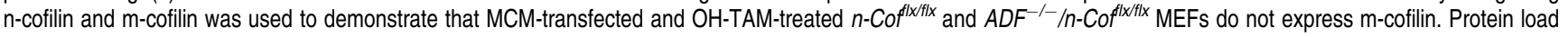
was controlled using a $\beta$-tubulin antibody

\begin{abstract}
Cytochrome $c$ release from mitochondria does not require ADF/cofilin activity. In a next series of experiments, we challenged mutant MEFs with wellcharacterized apoptosis-inducing agents and first measured mitochondrial release of the pro-apoptotic proteins cytochrome $c$ and SMAC. Although no cytochrome $c$ or SMAC was present in the cytosolic fractions of untreated control or mutant MEFs, both proteins were released upon STS exposure $(4 \mathrm{~h})$ in a manner similar to control MEFs, irrespective of the absence of $n$-cofilin, ADF, or both proteins (Figure 3a). Concordantly, the pro-apoptotic protein Bax translocated to mitochondria in all three mutant MEF lines. As $n$-cofilin was reported to translocate to mitochondria as early as $5 \mathrm{~min}$ after apoptosis induction, ${ }^{9}$ we investigated cytochrome $c$ release also during early phases of apoptosis (first $60 \mathrm{~min}$ ) and again found no differences between control and AC-MEFs (Figure 3b). Together, these results show that permeabilization of the mitochondrial outer membrane occurs independently of ADF/cofilin activity.
\end{abstract}

Apoptosis execution after exposure to STS in MEFs is unaltered in the absence of ADF/cofilin activity. We next asked whether the absence of n-cofilin and ADF might affect apoptosis signaling downstream of cytochrome $c$ release and examined the time course of caspase-3 activation, an essential step in the execution phases of apoptosis. Immunocytochemistry against active caspase-3 revealed similar amounts of active caspase-3-positive cells in controls and all three mutant MEF lines after $2 \mathrm{~h}$ or $4 \mathrm{~h}$ of STS exposure (Figures $4 a$ and b). Accordingly, western blotting against active caspase- 3 confirmed that caspase- 3 was activated to a similar extent in all four MEF lines (Figure 4c).

Nuclear condensation upon STS exposure does not require ADF/cofilin activity. To characterize apoptosis progression in mutant MEFs by an independent approach, we analyzed their nuclear morphology upon exposure to STS. Although untreated mutant MEFs displayed normal shapes and normal nuclear morphology, they underwent 
a

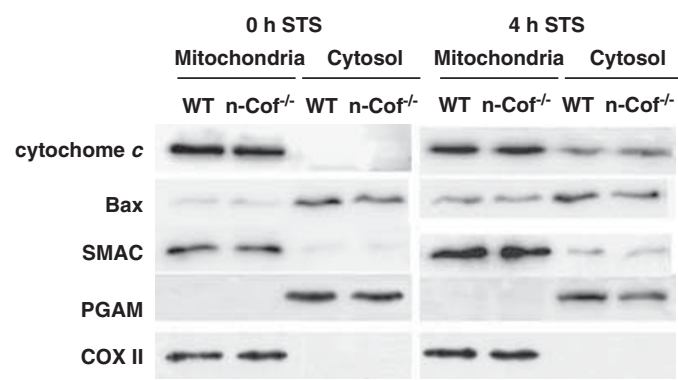

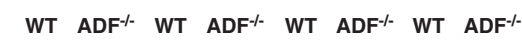
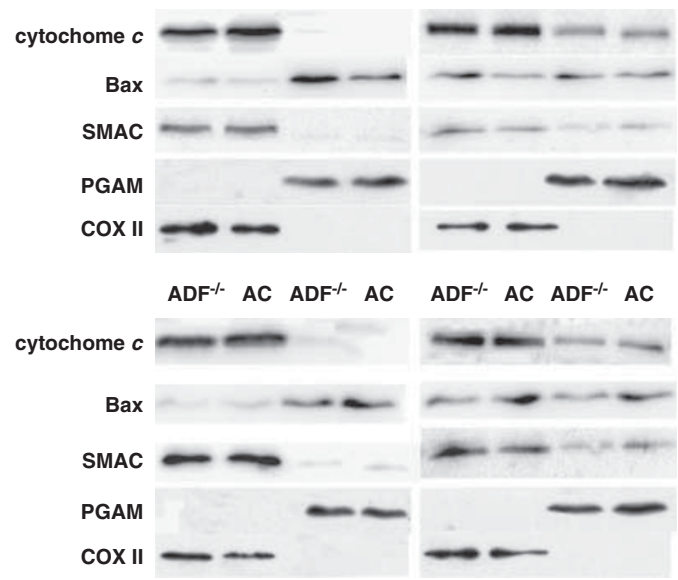

b STS $\frac{0}{\text { WT AC }} \frac{5}{\text { WT AC WT AC WT AC WT AC WT AC }} \frac{10}{\text { Win) }} \frac{30}{\text { WT }}$

cytochome $c$

Figure 3 Cytochrome $c$ and SMAC release from mitochondria does not require ADF/cofilin activity. (a) Mitochondrial release of cytochrome $c$ and SMAC and mitochondrial translocation of Bax were not altered in STS-treated MEFs deficient for n-cofilin (n-Cof $\left.{ }^{-/}\right)$, ADF $\left(A D F^{-/}\right)$, or both ADF/cofilin proteins (AC). Cytosolic and mitochondrial fraction of untreated and STS-treated MEFs was performed after digitonin permeabilization. (b) Cytosolic cytochrome $c$ levels were similar in WT- and AC-MEFs during the first hour of STS treatment. PGAM was used as a loading control

cellular and nuclear shrinkage, typical for apoptosis, upon exposure to STS (Figure 5a). Next, we quantified the relative numbers of apoptotic MEFs by scoring chromatin condensation and changes in nuclear morphology. At all five time points of STS treatment $(0.5-4 \mathrm{~h})$, chromatin condensation and nuclear morphology had changed in a manner that was indistinguishable between control and mutant MEFs (Figure 5b). Together, our data demonstrate that ADF/cofilin activity is not required for normal cytochrome $c$ release or caspase-3 activation, and that nuclear and cellular changes in morphology during apoptosis do not depend on the presence of ADF/cofilin.

Oxidant-triggered apoptosis proceeds normally in the absence of ADF/cofilin activity. N-cofilin has recently been linked to oxidant-induced apoptosis, as siRNAmediated knockdown of n-cofilin reportedly inhibits oxidantinduced cell death. ${ }^{10,12}$ We therefore wanted to test whether apoptosis, induced by the oxidant hydrogen peroxide
$\left(\mathrm{H}_{2} \mathrm{O}_{2}\right)$, is inhibited in MEFs devoid of any ADF/cofilin activity. Exposure of control MEFs to $\mathrm{H}_{2} \mathrm{O}_{2}$ induced a translocation of $n$-cofilin and ADF to mitochondria, concomitant with the release of cytochrome $c$ (Figure 6a). Importantly, genetic ablation of $n$-cofilin and/or ADF did not protect against oxidant-induced apoptosis, as apoptotic nuclear condensation proceeded normally in all mutant MEF lines (Figure 6b).

Activation of $n$-cofilin and ADF during apoptosis is essential for their mitochondrial translocation. We next aimed to unravel the mechanism by which $\mathrm{n}$-cofilin and ADF associate with mitochondria during apoptosis. We first investigated whether this is achieved by activities found in mitochondrial or cytosolic fractions of STS-treated cells. To do so, we performed reconstitution experiments with mitochondrial and cytosolic fractions from apoptotic or nonapoptotic control and AC-MEFs. These fractions were mixed in different combinations, and the association of $n$-cofilin and ADF with mitochondria was tested by co-fractionation upon centrifugation. As expected, we did not detect n-cofilin or ADF associated to mitochondria from non-apoptotic MEFs after incubation with the cytosol from non-apoptotic MEFs, thereby demonstrating successful separation of both protein fractions after incubation (Figure 7a, lanes 9 and 10). Instead, our analysis revealed a mitochondrial association of n-cofilin and ADF when mitochondria from non-apoptotic MEFs were incubated with the cytosolic fraction from apoptotic MEFs (lanes 7 and 8, as well as lanes 13 and 14). When mitochondria from apoptotic MEFs lacking $\mathrm{n}$-cofilin and ADF were incubated with the cytosolic fraction from non-apoptotic control MEFs, no mitochondrial translocation of $n$-cofilin and ADF occurred (lanes 11 and 12). Thus, our data show that changes within the cytosol, but not in mitochondria, mediate the mitochondrial translocation of $n$-cofilin and ADF during apoptosis.

Next, we investigated whether posttranslational modifications of $n$-cofilin and ADF occur during apoptosis and whether these modifications are crucial for their mitochondrial association. Activity of ADF/cofilin proteins is regulated via the phosphorylation state of a conserved serine residue at position 3 (Ser3). Ser3 dephosphorylation activates ADF/ cofilin and allows actin binding, whereas Ser3 phosphorylation inhibits ADF/cofilin activity. ${ }^{8}$ Using an antibody that specifically recognizes phosphorylated ADF/cofilin (phospho-AC), we found a significant reduction in phospho-AC levels upon STS treatment, whereas total $\mathrm{n}$-cofilin and ADF levels remained unchanged (Figures $7 \mathrm{~b}$ and c). By cell fractionation and western blotting, we did not find any phospho-AC associated with mitochondria, neither in untreated MEFs nor during STS-induced apoptosis (Figure 7d). Together, our data revealed ADF/cofilin dephosphorylation upon STS treatment, a step apparently crucial for the mitochondrial translocation of $n$-cofilin and ADF during apoptosis.

Mitochondrial translocation of $\mathrm{n}$-cofilin and ADF depends on actin. Dephosphorylated ADF/cofilin binds actin with high affinity, whereas the phosphorylated forms cannot interact. ${ }^{8}$ Because we only found dephosphorylated 


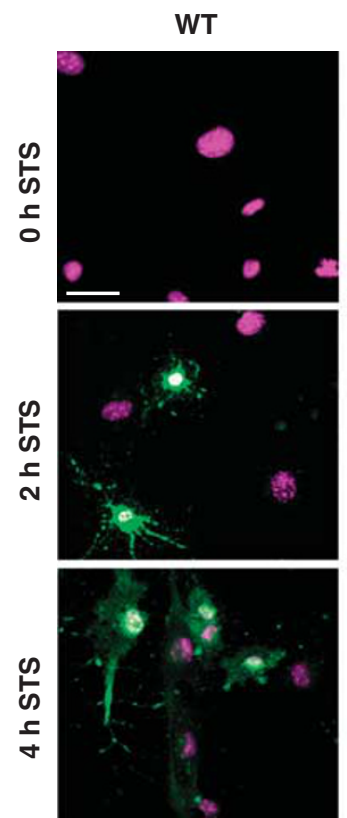

Hoechst/act. caspase-3
n-Cof ${ }^{-/-}$
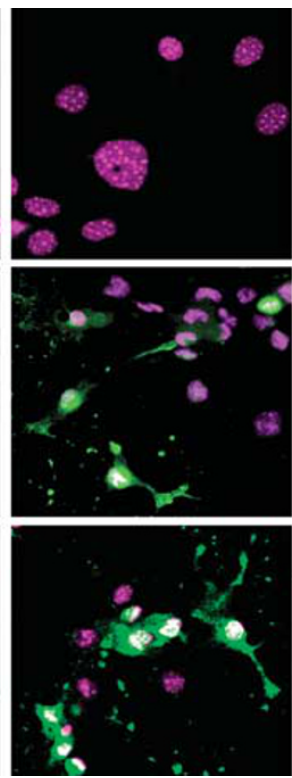

ADF $^{-/-}$
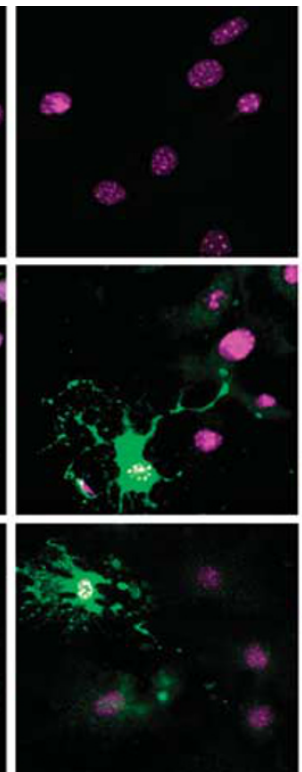

AC

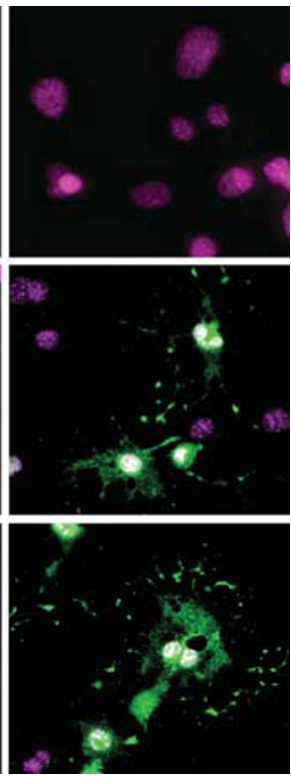

b

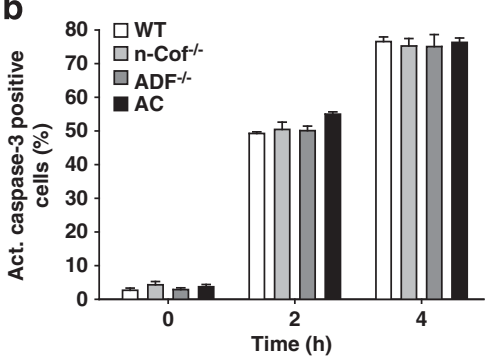

C
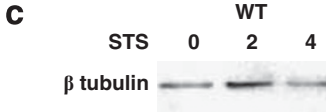

act. caspase-3

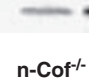

STS $\begin{array}{cccc}0 & 2 & 4\end{array}$

$\beta$ tubulin --

act. caspase-3

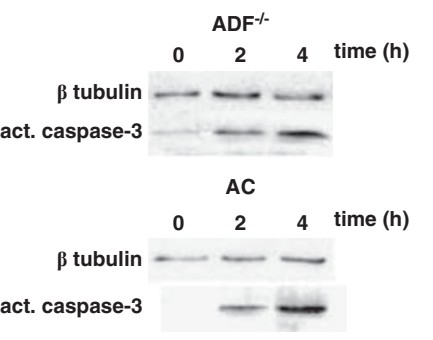

Figure 4 STS-induced activation of caspase-3 is unchanged in the absence of ADF/cofilin activity. (a) Activation of caspase-3 was unchanged in $n-\mathrm{Cof}^{-1-}, \mathrm{ADF}^{-1-}$, or AC-MEFs after 2 or $4 \mathrm{~h}$ of STS treatment. Caspase-3 activation was analyzed by immunofluorescence using an antibody against active caspase-3 (green). MEFs were counterstained with the nuclear marker Hoechst 33342 (magenta). Scale bar corresponds to $30 \mu \mathrm{m}$ in all panels. Representative images of six independent experiments are shown. (b) Quantification revealed similar percentages of active caspase-3-positive cells in all four MEF lines after 2 or $4 \mathrm{~h}$ of STS treatment. (c) Caspase-3 was activated similarly in all four MEF lines upon STS exposure. The cleaved fragment of activated caspase-3 was detected in whole-cell lysates using western blotting. Representative images of three independent experiments are shown

ADF/cofilin in mitochondrial fractions of apoptotic MEFs, we asked whether $n$-cofilin and ADF would recruit actin to mitochondria or whether $A D F / c o f i l i n$ translocate to mitochondria in an actin-dependent manner. Interestingly, we found actin present in the mitochondrial fraction from apoptotic MEFs, but not from non-apoptotic MEFs (Figure 8a). Concordantly, actin and mitochondria cofractionated on floatation gradients (Figure $8 b$ ). We next asked whether this association of actin with mitochondria depends on the presence of $n$-cofilin and ADF. We compared actin translocation with mitochondria in cells containing or lacking both ADF/cofilin proteins. Mitochondrial translocation of actin occurred similarly in the presence or absence of $A D F / c o f i l i n$ activity, demonstrating that actin translocates independent of $n$-cofilin or ADF to mitochondria during apoptosis (Figure 8c).

To test whether mitochondrial translocation of $n$-cofilin and ADF depends on actin, we incubated STS-treated MEF with jasplakinolide (JAS), a membrane-permeable peptide that specifically interacts with actin ${ }^{19}$ and inhibits actin-ADF/cofilin interaction. ${ }^{20}$ We found unchanged mitochondrial translocation of actin in JAS-treated apoptotic MEFs (Figure 8d). Conversely, JAS treatment significantly reduced the mitochondrial translocation of $\mathrm{n}$-cofilin and ADF in apoptotic MEFs, whereas cytochrome $c$ release was not affected. Importantly, JAS induced a dephosphorylation of ADF/cofilin in these cells, a finding reported for other cell lines before. ${ }^{21}$ Hence, we conclude that the reduced mitochondrial association of $n$-cofilin and ADF is the result of impaired actin interaction and not of elevated phospho-AC levels.

To investigate in more detail the dependence of this translocation on actin binding, we performed reconstitution experiments and found that the mitochondrial translocation of $n$-cofilin and ADF, but not of actin, was reduced by JAS when mitochondria from non-apoptotic MEFs were incubated with the cytosolic fraction from apoptotic MEFs (Figure 8e). Moreover, incubation of mitochondria from apoptotic MEFs with JAS reduced mitochondrial association of $n$-cofilin 
a
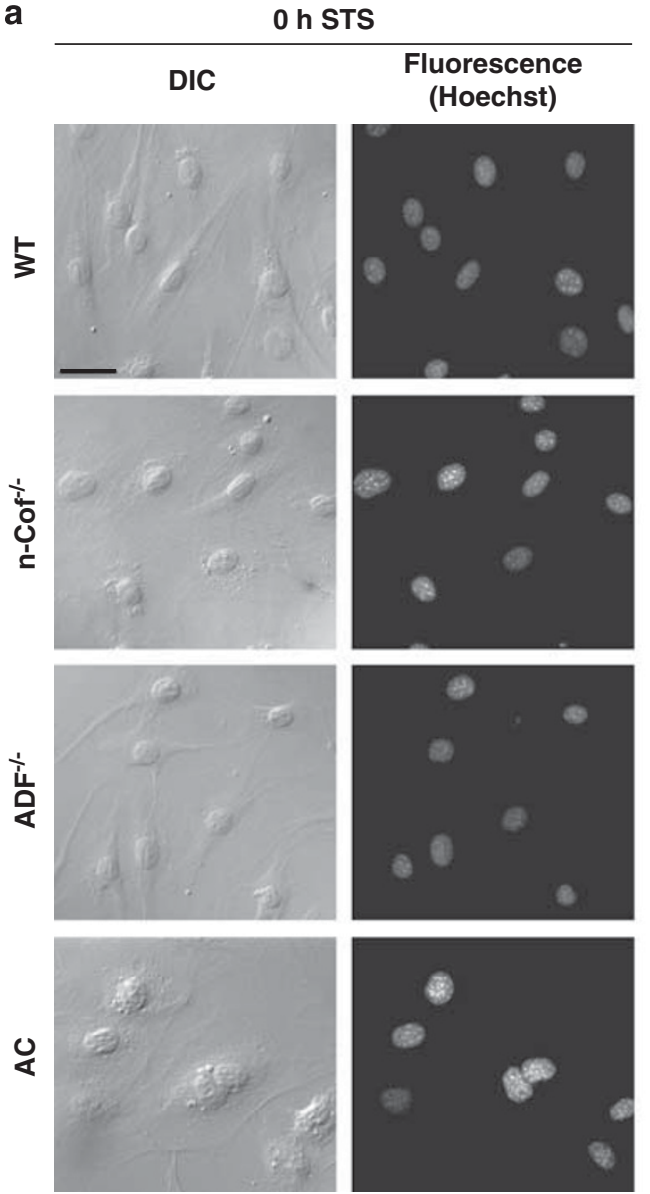

b

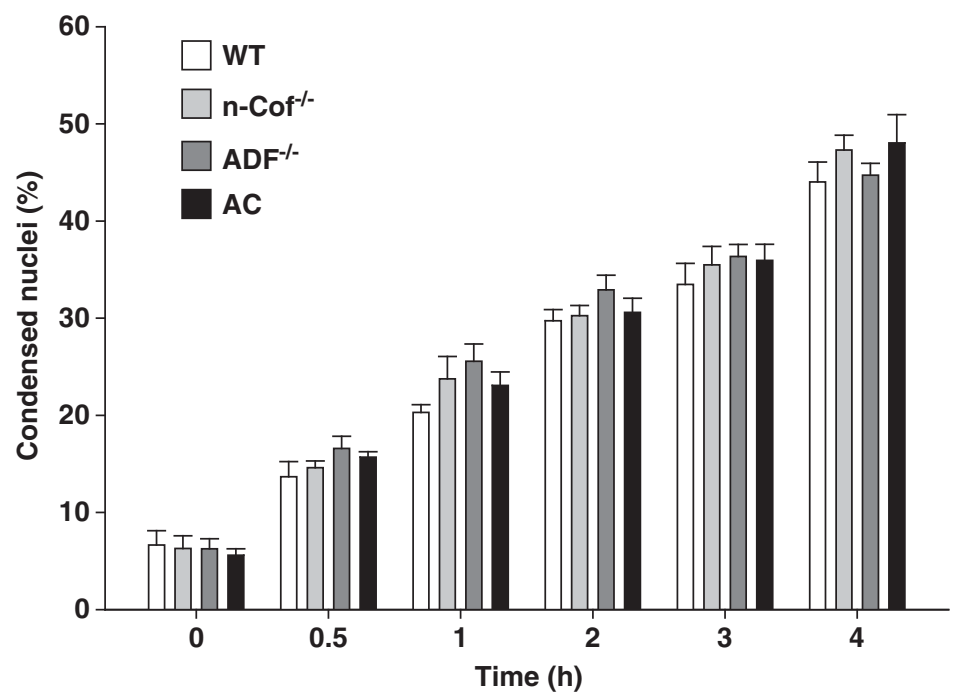

Figure 5 Nuclear condensation upon STS exposure does not require ADF/cofilin activity. (a) Time course of morphological changes of MEFs exposed to STS was similar in control and all three mutant MEF lines. Images were acquired with differential interference contrast microscopy (DIC), and nuclear morphology was determined using fluorescence microscopy employing DNA staining with Hoechst 33342. Thick arrows indicate apoptotic MEFs and thin arrows indicate non-apoptotic MEFs. Scale bar corresponds to $30 \mu \mathrm{m}$ in all panels. (b) STS-induced apoptosis at different time points $(0-4 \mathrm{~h})$ was scored according to chromatin condensation and nuclear morphology. Unaltered apoptosis rates were found in $n-\mathrm{Cof}^{-1-}, A D F^{-/-}$, or $\mathrm{AC}-\mathrm{MEFs}$ (quantified from six independent experiments)

and ADF, but not of actin (Figure 8f). To prove that pharmacological inhibition of actin-ADF/cofilin interaction is sufficient to inhibit ADF/cofilin translocation, we employed the membrane-impermeable bicyclic heptapeptide phalloidin (PHAL). Similar to JAS, PHAL inhibits interaction of actin with ADF/cofilin proteins. ${ }^{22,23}$ As seen with JAS, PHAL also 
a

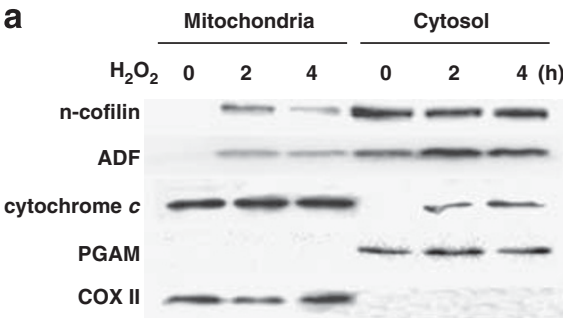

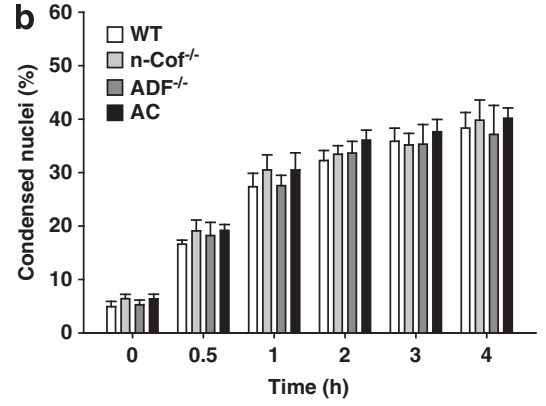

Figure 6 Normal $\mathrm{H}_{2} \mathrm{O}_{2}$-induced apoptosis in ADF/cofilin-deficient MEFs. (a) Translocation of $n$-cofilin and $A D F$ to the mitochondrial fraction during $\mathrm{H}_{2} \mathrm{O}_{2}$-induced apoptosis. Induction of apoptosis was confirmed by the translocation of cytochrome $c$ to the cytosol. (b) $\mathrm{H}_{2} \mathrm{O}_{2}$-induced apoptosis at different time points $(0-4 \mathrm{~h})$ was scored according to chromatin condensation and nucleus morphology. Unaltered apoptosis rates were found in $n-\mathrm{Cof}^{--}$, $A D F^{-/-}$, or AC-MEFs (quantified from six independent experiments)

inhibited mitochondrial translocation of $n$-cofilin and ADF in reconstitution experiments (Figure $8 \mathrm{e}$ ) and provoked a detachment of both ADF/cofilin proteins from mitochondria of apoptotic MEFs (Figure 8f). In both experiments, PHAL did not affect actin-mitochondria interaction. Together, we show that actin translocated to mitochondria during apoptosis and that this translocation preceded mitochondrial ADF/cofilin association. Our data suggest an indirect interaction of $n$ cofilin and ADF with mitochondria that depends on their actinbinding capacity and mitochondrially localized actin.

Concluding remarks. An important role of the actin depolymerizing protein $\mathrm{n}$-cofilin for apoptosis signaling was postulated recently, based on the findings that (i) $n$-cofilin translocates to mitochondria during apoptosis that in turn was necessary for (ii) cytochrome $c$ release, and that (iii) siRNA-mediated knockdown of n-cofilin expression conferred resistance to oxidant- or STS-induced apoptosis..$^{9,10}$ Using a genetically well-defined system of three MEF knockout lines, we report here that mitochondrial translocation of ADF/cofilin proteins does not represent an important step for cell death triggered by STS or $\mathrm{H}_{2} \mathrm{O}_{2}$ in these cells. The evidence for this is provided by the observation that genetic ablation of $n$ cofilin, ADF, or both ADF/cofilin proteins does not affect apoptosis signaling and execution. Specifically, we found that cytochrome $c$ and SMAC release from mitochondria, caspase- 3 activation, and nuclear chromatin condensation proceed normally in the absence of any ADF/cofilin activity. The observation that altered ADF/cofilin activity affects apoptosis progression in certain cell types ${ }^{9,10}$ suggests that cell-type-specific functions for ADF/cofilin in apoptosis signaling might exist. Accordingly, pharmacological intervention of actin dynamics impaired mitochondriamediated apoptosis in some cell types, but had no effect on apoptosis progression in others. ${ }^{5,24}$

Interestingly, our work confirmed that $n-$ cofilin $^{9,10}$ (as well as its homolog ADF, this work) translocates to mitochondria during apoptosis. Here, we reveal that this translocation is highly dependent on actin that also translocates to mitochondria in apoptotic MEFs. The mitochondrial translocation of actin during apoptosis is essential for the mitochondrial association of $n$-cofilin and ADF. ADF and $n$-cofilin undergo Ser3 dephosphorylation that enables these proteins to interact with cellular actin. ${ }^{8}$ As we found impaired mitochondrial association of $n$-cofilin and ADF in response to treatment with either JAS or PHAL, two peptides that block the interaction of $A D F /$ cofilin with actin, ${ }^{20,22-23}$ we demonstrate that mitochondrial association of $n$-cofilin and ADF is indirect and depends on mitochondrially localized actin.

Because our work revealed a translocation of actin to mitochondria during apoptosis, it is tempting to speculate that this interaction may be important for mitochondrial dynamics or distribution during cell death. It will be the subject of future research to unravel the molecular mechanism by which actin translocates to mitochondria and to test the significance of this process for cell death signaling.

\section{Materials and Methods}

Preparation and culture of MEFs. MEFs were isolated from wild-type controls (WT), ADF-null mice ( $\mathrm{ADF}^{-l-}$ ), conditional $n$-cofilin mutants ( $\mathrm{n}$-Cof $\left.\mathrm{f}^{\mathrm{f} / \mathrm{f} / \mathrm{fl})}\right),{ }^{16}$

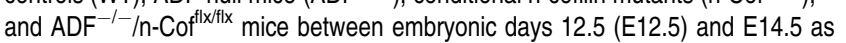
previously described. ${ }^{25}$ Briefly, dissected embryos were minced and treated with $0.25 \%$ trypsin (Invitrogen, Carlsbad, CA, USA) after removal of the head and organs. After $15 \mathrm{~min}$ of incubation at $37^{\circ} \mathrm{C}$, tissue was pulled through a $0.9-\mathrm{mm}$ needle to obtain isolated cells. After washing twice with serum-free Dulbecco's modified Eagle's medium (DMEM; Invitrogen), isolated cells were cultured in DMEM containing $10 \%$ fetal calf serum (FCS; PAA, Pasching, Austria). Primary MEFs (termed passage $0(P 0)$ ) were grown for 1 day. On the next day, MEF immortalization was started according to the 3-day transfer protocol (3T3) immortalization protocol that defined immortalization as completed after P12. MEFs were kept at $37^{\circ} \mathrm{C}$ and $5 \% \mathrm{CO}_{2}$ in DMEM containing $4.5 \mathrm{~g} / \mathrm{l}$ glucose and $10 \% \mathrm{FCS}$, as well as penicillin $(100 \mu / \mathrm{ml} ;$ PAA $)$ and streptomycin $(100 \mu \mathrm{g} / \mathrm{ml}$; PAA). MEFs were passaged every 2-3 days. All experiments were performed with MEFs of P12$\mathrm{P} 40$. For the induction of apoptosis, MEFs were treated with either $1 \mu \mathrm{M}$ STS (Sigma-Aldrich, St. Louis, MO, USA) or $200 \mu \mathrm{M} \mathrm{H}_{2} \mathrm{O}_{2}$ (Roth, Karlsruhe, Germany).

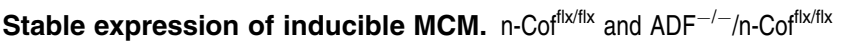
MEFs were transfected with a plasmid expressing MCM and puromycin acetyltransferase $^{18}$ by Fugene HD transfection reagent (Roche, Basel, Switzerland). Clones that stably expressed the MCM construct were isolated by $3 \mu \mathrm{g} / \mathrm{ml}$ puromycin (PAA) treatment. For the activation of MCM, n-Cof ${ }^{\text {flyflix }}$ and $\mathrm{ADF}^{-l-} / \mathrm{n}$-Cof ${ }^{|| x|f| x}$ MEFs were treated for $72 \mathrm{~h}$ with $1 \mu \mathrm{M}$ OH-TAM (Sigma-Aldrich).

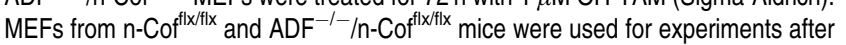
additional $24 \mathrm{~h}$ incubation in DMEM.

PCR. PCR was performed to verify MCM transfection and n-cofilin deletion upon OH-TAM treatment. For DNA extraction, MEFs were boiled for $60 \mathrm{~min}$ in a solution containing $25 \mathrm{mM} \mathrm{NaOH}$ and $0.2 \mathrm{mM} \mathrm{EDTA}$ (pH 12). Thereafter, the solution was neutralized by adding $40 \mathrm{mM}$ Tris- $\mathrm{HCl}(\mathrm{pH} 5)$, and debris was pelleted by a $10 \mathrm{~min}$ centrifugation step at $14000 \times g$. DNA-containing supernatant was used for PCR. 
Cre expression was confirmed by the amplification of a 726-bp fragment using the following primers: $5^{\prime}$-GTGGCAGATGGCGCGGCAACACCATT-3' and 5'-GCCTGC ATTACCGGTCGATGCAACGA-3'. Floxed (flx; $420 \mathrm{bp}$ ) and deleted $n$-cofilin gene (170 bp) were detected by a three-primer PCR using $5^{\prime}$-CGCTGGACCAGAGCA CGCGGCATC-3', 5'-CTGGAAGGGTTGTTACAACCCTGG-3' ${ }^{\prime}$, and 5' $5^{\prime}$-CATGAAG GTTCGCAAGTCCTCAAC- $3^{\prime}$.

MEF protein lysates. MEFs were lysed in $200 \mu$ l of ice-cold protein extraction buffer containing $150 \mathrm{mM} \mathrm{NaCl}, 50 \mathrm{mM}$ Tris- $\mathrm{HCl}(\mathrm{pH} 7.4), 2 \mathrm{mM}$ EDTA, and $0.5 \%$ Triton X-100. Protein lysates were homogenated by sonication. In some experiments, phosphatase inhibitor cocktail PhosSTOP (Roche) was added to detect phosphorylated ADF/cofilin (phospho-AC).

Preparation of mitochondrial and cytosolic fractions. MEF pellets were washed in ice-cold PBS and resuspended in $4 \mathrm{ml} \mathrm{MSH}$ buffer (mannitol-

a

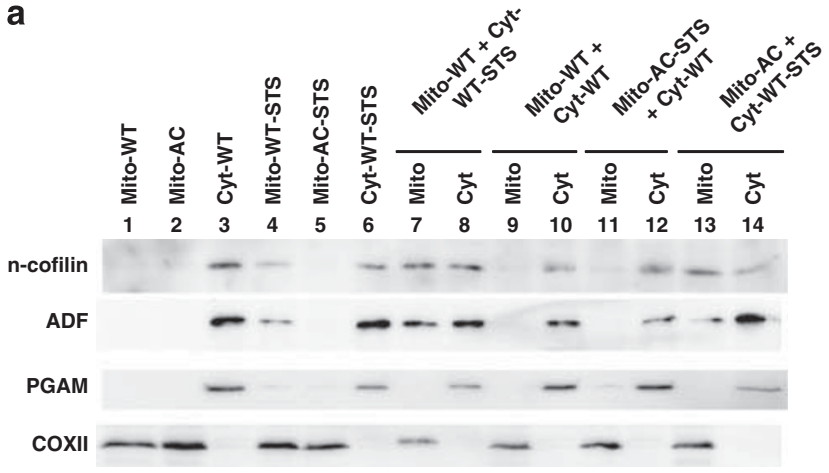

b

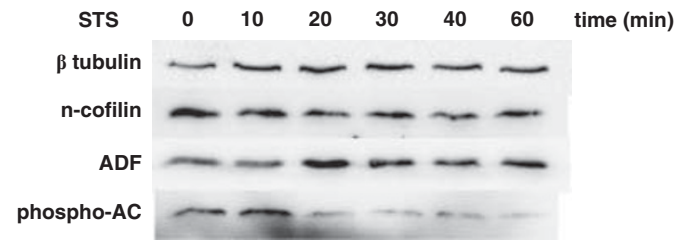

C

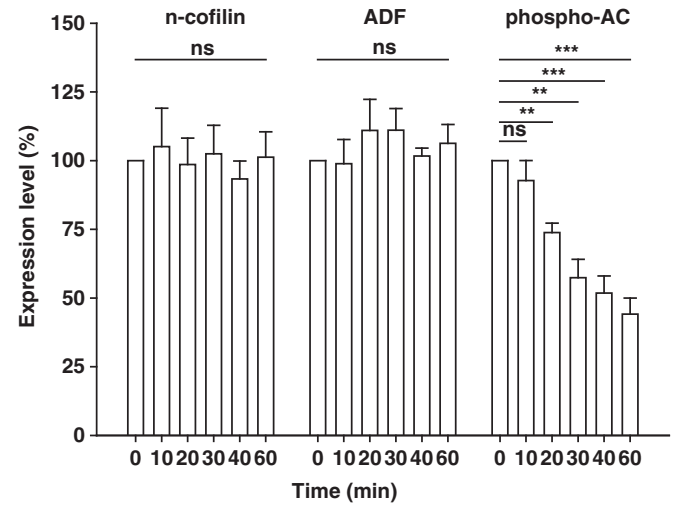

d

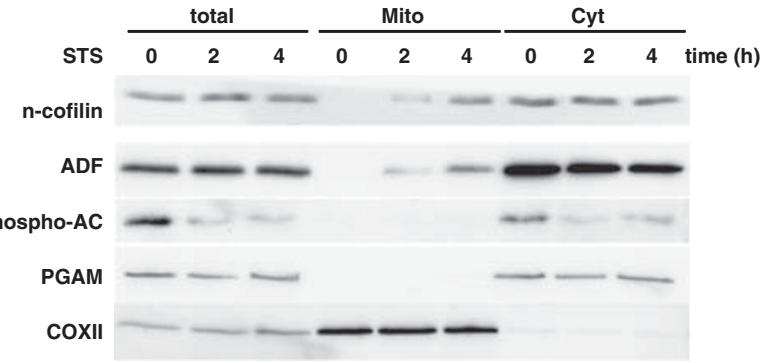

sucrose-hepes-buffer) containing: $210 \mathrm{mM}$ mannitol, $70 \mathrm{mM}$ sucrose, $5 \mathrm{mM}$ Tris$\mathrm{HCl}(\mathrm{pH} 7.5)$, and $1 \mathrm{mM}$ EDTA (pH 7.5). MEFs were homogenized using a tightfitting Douncer. Homogenates were centrifuged for $5 \mathrm{~min}$ at $4^{\circ} \mathrm{C}$ and $900 \times \mathrm{g}$, and the supernatant was centrifuged again for $15 \mathrm{~min}$ at $5500 \times g$. The supernatant contained the cytosolic proteins. For purification of mitochondria, the pellet was resuspended in MSH buffer and centrifuged for $15 \mathrm{~min}$ at $5500 \times \mathrm{g}$. Finally, the mitochondrial pellet was resuspended in MSH buffer at a protein concentration of $80-100 \mathrm{mg} / \mathrm{ml}$. Assays with isolated mitochondria were performed in MSH buffer. In all, 200-250 $\mu$ g mitochondria was incubated in samples of $100 \mu \mathrm{l} \mathrm{each} \mathrm{for} 30 \mathrm{~min}$ at $30^{\circ} \mathrm{C}$. Samples were centrifuged at $5500 \times g$ at $4^{\circ} \mathrm{C}$ for $15 \mathrm{~min}$ for separation of pellet and supernatant. The pellet was washed twice with MSH buffer to eliminate cytosolic contamination. In some experiments, JAS ( $3 \mathrm{mM}$; Calbiochem, Darmstadt, Germany) and PHAL (1 mM; Calbiochem) were used, as indicated in results and the figure legends. For the floatation assay, the mitochondrial fraction was adjusted to $2.1 \mathrm{M}$ sucrose. The 2.1-M sucrose suspension was overlaid with 1.8, 1.0, and $0.25 \mathrm{M}$ sucrose buffer containing $100 \mathrm{mM}$ Hepes and $100 \mathrm{mM}$ EGTA. The sample was centrifuged overnight at $40000 \times g$ in a Beckman swing rotor (SW 60, Beckman Coulter, Krefeld, Germany). The fractions were collected bottom-up using a gradient fractionator and further analyzed by SDS-PAGE.

Immunoblot. Protein lysates were mixed with SDS loading buffer containing $312 \mathrm{mM}$ Tris- $\mathrm{HCl}, 50 \mathrm{mM}$ DTT, $10 \%$ SDS, and $50 \%$ glycerine, boiled for $5 \mathrm{~min}$ and separated on $15 \%$ SDS gels. Protein load was controlled by Coomassie staining. Proteins were transferred to a PVDF membrane (Roth). Membranes were blocked for $1 \mathrm{~h}$ in T-TBS containing $150 \mathrm{mM} \mathrm{NaCl}, 20 \mathrm{mM}$ Tris- $\mathrm{HCl}(\mathrm{pH} 7.4)$, and $1 \%$ Tween-20 (Roth) supplemented with $5 \%$ dry milk. Incubation with primary antibody was performed in T-TBS $+5 \%$ dry milk. After washing, horseradish peroxidase (HRP)-conjugated secondary antibodies (anti-rabbit IgG-HRP (Pierce, Rockford, IL, USA), anti-mouse IgG-HRP (Pierce), or anti-goat IgG-HRP (Molecular Probes, Grand Island, NY, USA)) were added at a concentration of 1:5000. Subsequently, membranes were washed and incubated in ECL Western blotting detection reagent (Amersham Biosciences, Munich, Germany). The following primary antibodies were used: mouse anti-actin (1: 1000; Abcam, Cambridge, UK), mouse anti-ADF (1:5000; clone GV13; Sigma-Aldrich), rabbit anti-Bax (1:1000; Cell Signaling Technology, Danvers, MA, USA), rabbit anti-activated caspase-3 (1:1000; Cell Signaling Technology), mouse anti-cytochrome $c(1: 500$; clone 7H8.2C12; BD Pharmingen, Franklin Lakes, NJ, USA), goat anti-phosphoglycerate mutase (PGAM; 1: 1000; Santa Cruz Biotechnology, Santa Cruz, CA, USA), rabbit anti-phospho-AC (1: 1000; Cell Signaling Technology), rabbit anti-SMAC (1: 1000; Sigma-Aldrich), and mouse anti- $\beta$-tubulin (1:1000; clone TUB 2.1; Sigma-Aldrich). Rabbit anti-cytochrome $c$ oxidase subunit II (COX II) antibody (1:1000) was generated by immunizing rabbits with recombinantly expressed and purified mouse COX II protein. Generation of the rabbit anti-n-cofilin (KG 40) and the rabbit antibody that recognizes n-cofilin and m-cofilin (KG 60) was described before. ${ }^{16}$

Figure 7 Activation of $n$-cofilin and ADF during apoptosis is essential for their mitochondrial translocation. (a) Mitochondria from non-apoptotic control MEFs (Mito-WT) and non-apoptotic ADF- and n-cofilin-deficient MEFs (Mito-AC) or from apoptotic control and AC-MEFs (Mito-WT-STS and Mito-AC-STS, respectively) were incubated with cytosolic fraction from either non-apoptotic (Cyt-WT) or apoptotic control MEF (Cyt-WT-STS). Mitochondrial translocation of $n$-cofilin and ADF was observed when mitochondria from non-apoptotic control MEFs were incubated with the cytosolic fraction from apoptotic control MEFs (lanes 7 and 8 , as well as 13 and 14), but not when mitochondria from apoptotic MEFs were incubated with the cytosolic fraction from non-apoptotic MEFs (lanes 11 and 12). Separation of mitochondrial and cytosolic fractions was controlled with antibodies against PGAM and COX II. Lanes 1-6 indicate in which of the protein fractions n-cofilin and ADF were present. (b) Western blots demonstrating reduction of Ser3 phosphorylation of $A D F / c o f i l i n$ (phospho-AC) upon STS treatment. No changes were found for total $n$-cofilin or ADF levels. Equal loading was controlled by analyzing $\beta$-tubulin levels. (c) Quantification of $n$-cofilin, ADF, and phospho-AC levels in STS-treated MEFs. No significant change was found for n-cofilin or ADF, whereas phospho-AC levels were reduced to $57.4 \pm 6.7 \%$ of controls (untreated MEFs) 30 min after STS treatment $(n=6 ; P<0.01)$ and to $44.1 \pm 5.8 \% 60 \mathrm{~min}$ after STS treatment $(n=6 ; P<0.01)$. (d) Western blots demonstrating that $n$-cofilin and ADF, but not phospho-AC, translocate to mitochondria upon STS treatment. PGAM and COX II antibodies prove successful separation of mitochondria and cytosol 
a

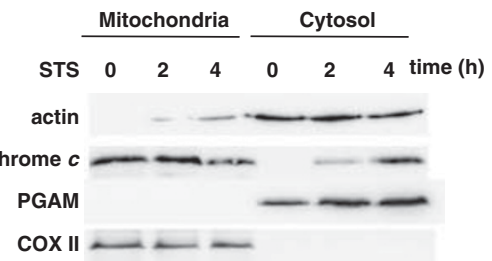

b

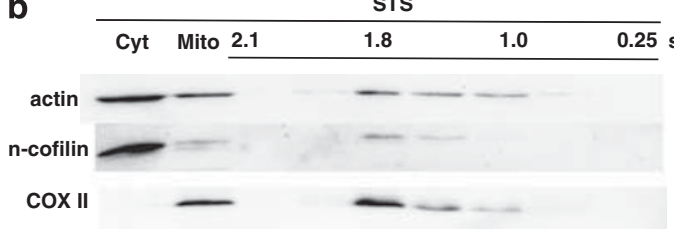

C

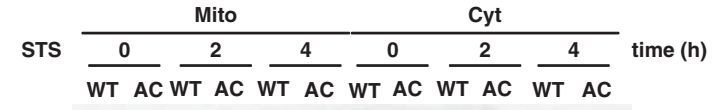

$$
\begin{aligned}
& \text { actin } \quad-\cdots-\infty-\cdots
\end{aligned}
$$

PGAM

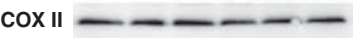

d

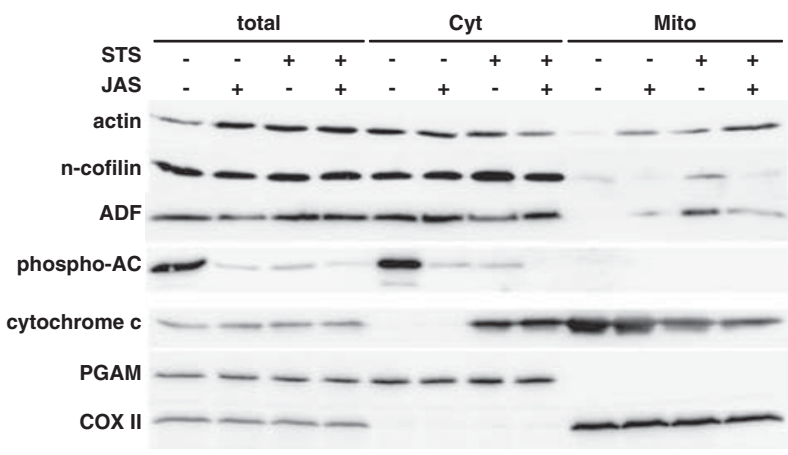

e
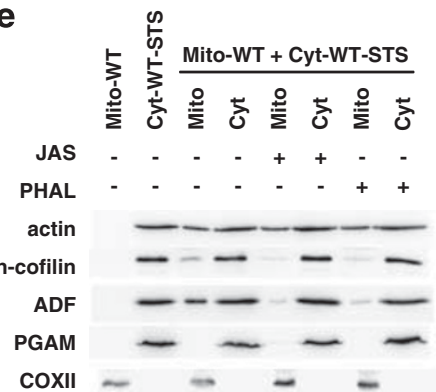

f

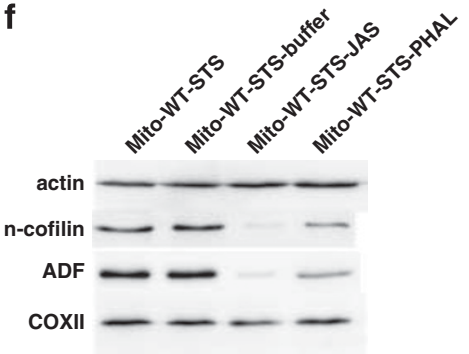

Figure 8 STS-induced mitochondrial translocation of n-cofilin and ADF is mediated by actin. (a) Translocation of actin to mitochondria during STS-induced apoptosis. Induction of apoptosis was confirmed by cytochrome $c$ release. After harvesting, untreated and STS-treated MEFs were separated into a cytosolic and a mitochondrial fraction. (b) Association of actin with mitochondria. Mitochondrial fractions from STS-treated MEFs were fractionated using floatation centrifugation. Western blot analysis of protein fractions revealed co-migration of actin with n-cofilin and mitochondria. (c) Independence of mitochondrial translocation of actin from n-cofilin and ADF. Similar actin levels were found in mitochondrial fractions of STS-treated control (WT) and AC-MEFs that were deficient for n-cofilin and ADF. (d) Dependence of mitochondrial translocation of n-cofilin and ADF on actin. Treatment of apoptotic MEFs with JAS, which blocks actin-ADF/cofilin interaction, did not alter mitochondrial translocation of actin, but dephosphorylated ADF/cofilin and reduced n-cofilin and ADF levels in mitochondrial fractions. (e) JAS and PHAL, a second peptide that blocks actin-ADF/cofilin interaction, reduced the mitochondrial translocation of $n$-cofilin and ADF in reconstitution experiments, in which mitochondria from non-apoptotic control MEFs (Mito-WT) were incubated with the cytosolic fraction from apoptotic control MEFs (Cyt-WT-STS). Conversely, JAS and PHAL had no effect on the mitochondrial translocation of actin. (f) Similarly, JAS and PHAL reduced the association of n-cofilin and ADF with mitochondria from apoptotic MEFs. Again, both peptides did not influence the interaction of actin with mitochondria

Release of cytochrome $c$ and SMAC. MEFs were pelleted and washed in PBS. MEFs $\left(25 \times 10^{6}\right.$ cells $\left./ \mathrm{ml}\right)$ were incubated for $60 \mathrm{~min}$ at $30^{\circ} \mathrm{C}$ in cytosol fractionation buffer containing $20 \mathrm{mM}$ Hepes $(\mathrm{pH} 7.4), 5 \mathrm{mM} \mathrm{MgCl} 2,10 \mathrm{mM} \mathrm{KCl}$, $1 \mathrm{mM}$ EDTA, $1 \mathrm{mM}$ EGTA, and $0.02 \%$ (w/v) digitonin. MEFs were fractionated by centrifugation for $15 \mathrm{~min}$ at $16000 \times \mathrm{g}$ and $4^{\circ} \mathrm{C}$. The supernatant contained cytosolic proteins, whereas mitochondrial proteins were present in the pellet. Each set of experiments was repeated four times.

Immunocytochemistry. Roughly $80000 \mathrm{MEFs}$ were seeded on $12 \mathrm{~mm}$ coverslips. MEFs were fixed for $15 \mathrm{~min}$ in $4 \%$ paraformaldehyde at $4{ }^{\circ} \mathrm{C}$ and permeabilized by a 1 -h incubation in $0.3 \%$ Triton X-100, $10 \mathrm{mM}$ Hepes (pH 7.4), $3 \%$
BSA, and $0.1 \% \mathrm{NaN}_{3}$. MEFs were stained with an antibody against activated caspase-3 (1:500; Cell Signaling Technology) overnight at $4^{\circ} \mathrm{C}$, followed by the incubation with appropriate Alexa Fluor 488-conjugated secondary antibody (1:1000; Molecular Probes) for $1 \mathrm{~h}$ at RT. After washing, MEFs were stained with $1 \mu \mathrm{g} / \mathrm{ml}$ Hoechst 33342 (Molecular Probes) for $30 \mathrm{~min}$ and mounted with a homemade mounting solution based on glycerol, polyvinyl alcohol, and DABCO. Apoptotic cells were identified by nuclear condensation and DNA fragmentation visualized by fluorescence microscopy using a Zeiss Axioskop 2 microscope (Carl Zeiss, Göttingen, Germany), an F-View II camera (Münster, Germany), and CellF imaging software (Olympus, Hamburg, Germany). Each set of experiments was repeated six times, with at least $300 \mathrm{MEFs}$ counted in each experiment. 
Statistics. Sets of data are presented by their mean values and standard error of the means. For statistical analysis, an unpaired two-tailed Student's $t$-test was performed using Microsoft Excel software.

\section{Conflict of Interest}

The authors declare no conflict of interest.

Acknowledgements. We thank Dr. W Witke (Bonn/Germany) for providing ADF and n-Cof ${ }^{|1 / f| x}$ mice, Dr. JM Herrmann (Kaiserslautern/Germany) for support, Dr. M Reth (Freiburg/Germany) for providing the MCM cassette, and Drs S Orrenius and B Zhivotovsky (Stockholm/Sweden) for critical reading the manuscript. This work was funded by the Research Initiative Membrane Transport (RIMB) of the University of Kaiserslautern. KR is a recipient of a doctoral fellowship from the Stipendienstiftung des Landes Rheinland-Pfalz.

\section{Author Contributions}

Experiments were designed and results were discussed by KR, MO, and MBR. Mutant mice were generated by CBG. MEFs were generated by KR and MC. Data analysis was performed by KR. Manuscript was written by MO, EF, and MBR.

1. Ziegler DS, Kung AL. Therapeutic targeting of apoptosis pathways in cancer. Curr Opin Oncol 2008; 20: 97-103.

2. Okouchi M, Ekshyyan O, Maracine M, Aw TY. Neuronal apoptosis in neurodegeneration. Antioxid Redox Signal 2007; 9: 1059-1096.

3. Wang $X$. The expanding role of mitochondria in apoptosis. Genes Dev 2001; 15 : 2922-2933.

4. Liu X, Kim CN, Yang J, Jemmerson R, Wang X. Induction of apoptotic program in cell-free extracts: requirement for dATP and cytochrome c. Cell 1996; 86: 147-157.

5. Gourlay CW, Ayscough KR. The actin cytoskeleton: a key regulator of apoptosis and ageing? Nat Rev Mol Cell Biol 2005; 6: 583-589.

6. Anesti V, Scorrano $\mathrm{L}$. The relationship between mitochondrial shape and function and the cytoskeleton. Biochim Biophys Acta 2006; 1757: 692-699.

7. Ott M, Gogvadze V, Orrenius S, Zhivotovsky B. Mitochondria, oxidative stress and cell death. Apoptosis 2007; 12: 913-922.

8. Bamburg JR, Wiggan OP. ADF/cofilin and actin dynamics in disease. Trends Cell Biol 2002; 12: 598-605.

9. Chua BT, Volbracht C, Tan KO, Li R, Yu VC, Li P. Mitochondrial translocation of cofilin is an early step in apoptosis induction. Nat Cell Biol 2003; 5: 1083-1089.
10. Klamt F, Zdanov S, Levine RL, Pariser A, Zhang Y, Zhang B et al. Oxidant-induced apoptosis is mediated by oxidation of the actin-regulatory protein cofilin. Nat Cell Biol 2009; 11: 1241-1246.

11. Zhu B, Fukada K, Zhu H, Kyprianou N. Prohibitin and cofilin are intracellular effectors of transforming growth factor beta signaling in human prostate cancer cells. Cancer Res 2006; 66: 8640-8647.

12. Wabnitz GH, Goursot $C$, Jahraus B, Kirchgessner $H$, Hellwig A, Klemke $M$ et al. Mitochondrial translocation of oxidized cofilin induces caspase-independent necrotic-like programmed cell death of T cells. Cell Death Dis 2010; 1 : e58.

13. Field JM. The actin cytoskeleton and cell survival. In: Anninos P, Rossi M, Pham TD, Falugi, Bussing A, Koukkou M (eds). Recent Advances in Clinical Medicine. WSEAS Press: Cambridge, UK, 2010, pp 322-330.

14. Rust MB, Gurniak CB, Renner M, Vara H, Morando L, Gorlich A. et al. Learning, AMPA receptor mobility and synaptic plasticity depend on $n$-cofilin-mediated actin dynamics. EMBO J 2010; 29: 1889-1902.

15. Görlich A, Wolf M, Zimmermann AM, Gurniak CB, Al Banchaabouchi M, Sassoe-Pognetto $\mathrm{M}$ et al. $\mathrm{N}$-cofilin can compensate for the loss of ADF in excitatory synapses. PLoS One 2011; 6: e26789.

16. Bellenchi GC, Gurniak CB, Perlas E, Middei S, Ammassari-Teule M, Witke W. N-cofilin is associated with neuronal migration disorders and cell cycle control in the cerebral cortex. Genes Dev 2007; 21: 2347-2357.

17. Gurniak $\mathrm{CB}$, Perlas $\mathrm{E}$, Witke W. The actin depolymerizing factor n-cofilin is essential for neural tube morphogenesis and neural crest cell migration. Dev Biol 2005; 278: 231-241.

18. Verrou C, Zhang Y, Zürn C, Schamel WW, Reth M. Comparison of the tamoxifen regulated chimeric Cre recombinases MerCreMer and CreMer. Biol Chem 1999; 380: 1435-1438.

19. Holzinger A. Jasplakinolide: an actin-specific reagent that promotes actin polymerization. In: Gavin RH (ed). Cytoskeleton Methods and Protocols, Methods in Molecular Biology. Humana Press: Totowa, NJ, USA, 2009, pp 71-87.

20. Tsuji T, Miyoshi T, Higashida C, Narumiya S, Watanabe N. An order of magnitude faster AIP1-associated actin disruption than nucleation by the Arp2/3 complex in lamellipodia. PloS One 2009; 4: e4921.

21. Jovceva E, Larsen MR, Waterfield MD, Baum B, Timms JF. Dynamic cofilin phosphorylation in the control of lamellipodial actin homeostasis. J Cell Sci 2007; 120: 1888-1897.

22. Hayden SM, Miller PS, Brauweiler A, Bamburg JR. Analysis of the interactions of actin depolymerizing factor with G- and F-actin. Biochemistry 1993; 32: 9994-10004.

23. Ressad F, Didry D, Xia GX, Hong Y, Chua NH, Pantaloni D et al. Kinetic analysis of the interaction of actin-depolymerizing factor (ADF)/cofilin with G- and F-actins. Comparison of plant and human ADFs and effect of phosphorylation. J Biol Chem 1998; 273: 20894-20902.

24. Okada C, Sanders ML, Crews P. Jasplakinolide induces apoptosis in various transformed cell lines by a caspase-3-like protease-dependent pathway. Clin Diagn Lab Immunol 2000; 7: 947-952.

25. Seiler A, Schneider M, Forster H, Roth S, Wirth EK, Culmsee C et al. Glutathione peroxidase 4 senses and translates oxidative stress into 12/15-lipoxygenase dependentand AIF-mediated cell death. Cell Metab 2008; 8: 237-248. 